\title{
Short circuit current improvement in planar heterojunction organic solar cells by multijunction charge transfer
}

\author{
J. C. Wang, ${ }^{1}$ S. Q. Shi, ${ }^{1, a)}$ C. W. Leung, ${ }^{2}$ S. P. Lau, ${ }^{2}$ K. Y. Wong, ${ }^{3}$ and P. K. L. Chan ${ }^{1,4, a)}$ \\ ${ }^{1}$ Department of Mechanical Engineering, The Hong Kong Polytechnic University, Hong Kong \\ ${ }^{2}$ Department of Applied Physics, The Hong Kong Polytechnic University, Hong Kong \\ ${ }^{3}$ Department of Applied Biology and Chemical Technology, The Hong Kong Polytechnic University, \\ Hong Kong \\ ${ }^{4}$ Department of Mechanical Engineering, The University of Hong Kong, Hong Kong
}

(Received 5 November 2011; accepted 4 January 2012; published online 30 January 2012)

\begin{abstract}
A multijunction structure was applied on an organic photovoltaic (OPV) device for broadening the absorption spectrum and enhancing the power conversion efficiency through charge transfer process. By inserting the tris[4-(2-thienyl)]amine (TTPA) into a boron subphthalocyanine chloride $(\mathrm{SubPc}) / \mathrm{C}_{60}$ OPV device, the short circuit current density $\left(\mathrm{J}_{\mathrm{sc}}\right)$ showed a $47.5 \%$ increases from 3.05 to $4.50 \mathrm{~mA} / \mathrm{cm}^{2}$ in the bilayer planar heterojunction device, while the open circuit voltage $\left(\mathrm{V}_{\mathrm{oc}}\right)$ remained constant. Based on the single junction (TTPA/SubPc) device and photoluminescence absorption results, we confirmed both TTPA/SubPc and $\mathrm{SubPc} / \mathrm{C}_{60}$ junctions are contributing to the exciton dissociation process hence the efficiency enhancement. (c) 2012 American Institute of Physics. [doi:10.1063/1.3680253]
\end{abstract}

One of the main factors which limit the performance of earlier organic photovoltaic (OPV) was the narrow optical absorption range when compared with silicon-based devices which limit the number of free changes generation. To overcome this, for example, in the dye sensitized solar cells (DSSCs), the absorption is enhanced by adding second absorber or dye into the device where the energy is transferred by Förster resonant energy transfer (FRET). ${ }^{1-4}$ This process involves dipole-dipole coupling of donor and acceptor by an electric field and strongly relies on the overlapping between donor emission and acceptor absorption spectra as well as the distance between the donor-acceptor separation distances $(d) .{ }^{2,3}$ However, due to the large separation distance between the donor and acceptor materials, the FRET process is unfavorable in small molecular weight planar heterojunction (PHJ) OPV devise. One of the possible approaches to overcome the absorption limit in PHJ solar cell is make use of the tandem structure in which individual solar cells are connected together by the intermediate recombination layers. ${ }^{5,6}$ In the tandem structure device, the photogenerated currents in each individual cells should be matched for preventing the charge accumulation inside the device hence reducing the overall short circuit current density output $\left(\mathrm{J}_{\mathrm{sc}}\right)$. Furthermore, the thicknesses of the recombination layers should be designed for effective charge collection and have minimum blockage of the incident light. ${ }^{7}$ To overcome these fabrication challenges, the structure optimization for high efficiency tandem OPV is a very popular research area.

In the current work, we demonstrated that the performance of the bi-layer OPV can be enhanced by placing a second photo-absorber (donor) into the device and forming multijunctions without the tandem structure. Through inserting

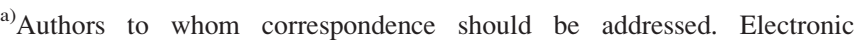
addresses: mmsqshi@polyu.edu.hk (Tel.: 852 27667821) and pklc@hku.hk (Tel.: 852 28592634).
}

the tris[4-(2-thienyl)]amine (TTPA) into a boron subphthalocyanine chloride $(\mathrm{SubPc}) / \mathrm{C}_{60}$ bilayer single planar heterojunction solar cell, we improved the $\mathrm{J}_{\mathrm{sc}}$ value for $47.5 \%$ from 3.05 to $4.50 \mathrm{~mA} / \mathrm{cm}^{2}$, while keeping the $\mathrm{V}_{\mathrm{oc}}$ unchanged. We also performed photoluminescence (PL) absorption measurements for different combination of the thin films and examined the J-V curves of single junction TTPA/ SubPc device. Based on the results, we conclude that the enhancement of the tri-layer OPV are attributed to (1) the complement of the optical absorption from the TTPA and SubPc layers and (2) two heterojunctions for exciton dissociation and the favorable energy level alignment for efficient charge transfer (CT). The key parameters in designing the structure of the multijunction planar organic solar cells are also discussed.

The schematic diagram of the device structure, the corresponding energy diagrams of the multijunction OPV and the chemical structure of TTPA and SubPc are shown in Fig. 1(a). For the fabrication and experimental details, see supplementary material. ${ }^{15}$ In all the devices, $10 \mathrm{~nm}$ of bathocuproine (BCP) is used for the hole blocking and $100 \mathrm{~nm}$ of $\mathrm{Ag}$ is used as the cathode ${ }^{8}$ (detailed description of the device fabrication can be found in the supplementary information). The band structure of the multijucntion device in Fig. 1(a) is similar to the cascade energy structure formed by placing a very thin intermediate layer between the donor and acceptor reported before for the short circuit current improvement. However, the main drawback of the cascade structure is the decreases of the open circuit voltage while the value of the $\mathrm{J}_{\mathrm{sc}}$ increases. ${ }^{9-11}$ Furthermore, the thin intermediate layer (less than $5 \mathrm{~nm}$ ) used in the cascade structure also limits the photon absorption and thus the exciton generation in the device. $^{10,11}$ Different from the conventional cascade structure OPV, the TTPA layer applied in the current multijunction device has $20 \mathrm{~nm}$ thickness for optical absorption and it also has similar highest occupied molecular orbital (HOMO) level as the SubPc layer. ${ }^{12}$ From the J-V curves of the 
(a)
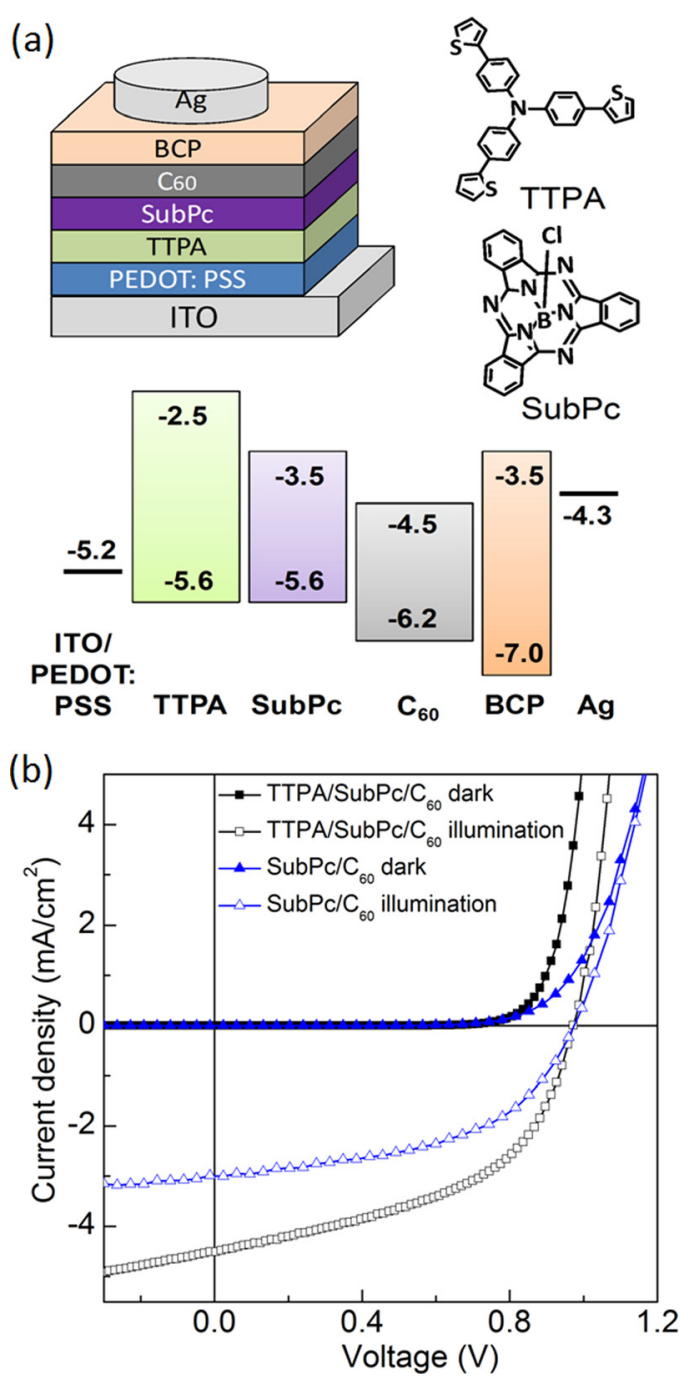

FIG. 1. (Color online) (a) The schematic diagram of the multijunction device and the corresponding energy diagram. (b) The J-V curves multijunction device with the TTPA layer: ITO/PEDOT:PSS/TTPA $(20 \mathrm{~nm}) /$ $\operatorname{SubPc}(13 \mathrm{~nm}) / \mathrm{C}_{60}(32.5 \mathrm{~nm}) / \mathrm{BCP}(10 \mathrm{~nm}) / \mathrm{Ag}(100 \mathrm{~nm})$ and single junction reference device without the TTPA layer: ITO/PEDOT:PSS/SubPc $(13 \mathrm{~nm}) /$ $\mathrm{C}_{60}(32.5 \mathrm{~nm}) / \mathrm{BCP}(10 \mathrm{~nm}) / \mathrm{Ag}(100 \mathrm{~nm})$.

multijunction and single junction devices in Fig. 1(b), we noticed the band alignment of the multijucntion device allows the open circuit voltage to remain almost unchanged while the $\mathrm{J}_{\mathrm{sc}}$ increases from $3.05 \mathrm{~mA} / \mathrm{cm}^{2}$ to $4.5 \mathrm{~mA} / \mathrm{cm}^{2}$ and resulting in the overall peak-to-correlation energy (PCE) increases from $1.49 \%$ to $2.18 \%$. Although this value of efficiency is not the highest value for planar heterojunction $\mathrm{OPV}$, the improvement in $\mathrm{J}_{\mathrm{sc}}$ without the decay of $\mathrm{V}_{\mathrm{oc}}$ in the current multijunction device provides a direction for the efficiency improvement without complex tandem structure. In the following section, we will focus on investigating the physical mechanism for the efficiency enhancement.

Fig. 2(a) shows the absorbance and the external quantum efficiency (EQE) of multijunction device as well as the reference bilayer $\mathrm{SubPc} / \mathrm{C}_{60}$ device. The increase of the optical absorption of the complex film and the EQE at the range of around $340 \mathrm{~nm}$ to $440 \mathrm{~nm}$ is attributed to the presence of the TTPA thin film which can absorb high energy photons for exciton generation. It is important to notice that the lower energy photon quantum yield rate from the SubPc is not affected by the presence of the TTPA layer, which suggests

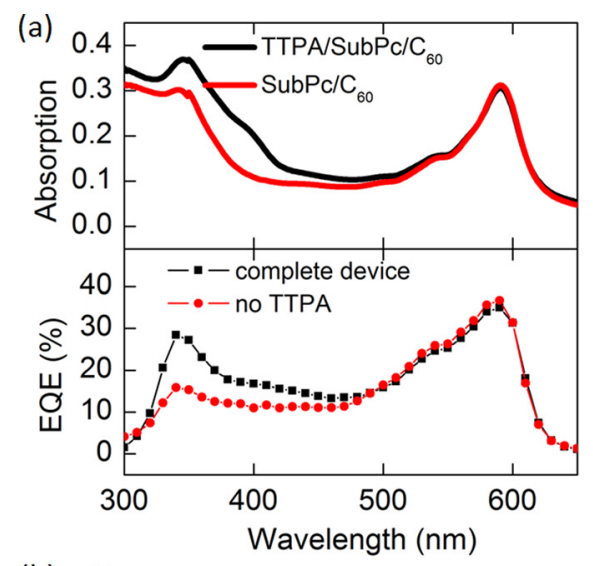

(b)

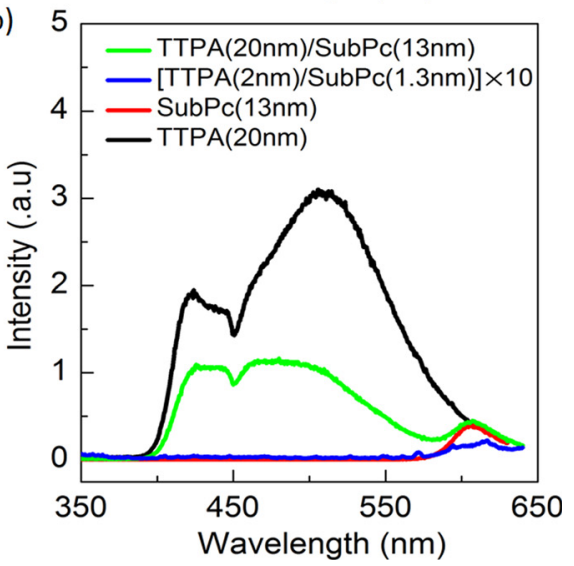

FIG. 2. (Color online) (a) The absorbance profiles of the complete active layers comparing with the reference and the EQE of the multijunction device in comparing with reference device. (b) The PL of the organic semiconductor films for a pump wavelength of $340 \mathrm{~nm}$ at room temperature.

the TTPA layer generate extra free carriers. To investigate the dissociation location of the excitons for these extra carriers, we performed room temperature PL measurement on four various samples: bilayer $\operatorname{TTPA}(20 \mathrm{~nm}) / \operatorname{SubPc}(13 \mathrm{~nm})$, multi-layer $[\operatorname{TTPA}(2 \mathrm{~nm}) / \operatorname{SubPc}(1.3 \mathrm{~nm})] \times 10$, single layer $\operatorname{SubPc}(13 \mathrm{~nm})$, and single layer TTPA $(20 \mathrm{~nm})$. In the PL results shown in Fig. 2(b), the bilayer film with TTPA and SubPc (green curve) shows a significant drop at the TTPA emission peaks when compared with the single TTPA layer (black curve). It indicates the excitons in the TTPA layer were quenched by the presence of the TTPA/SubPc interface. The dropping of the PL peaks is more obvious in multilayer film which consists 10 periods of TTPA $(2 \mathrm{~nm}) /$ $\operatorname{SubPc}(1.3 \mathrm{~nm})$ (total thicknesses of the TTPA and SubPc films are remaining at $20 \mathrm{~nm}$ and $13 \mathrm{~nm}$, respectively) where the TTPA peaks are completely vanished. It is worth to mention the exciton transfer from the TTPA layer to the SubPc layer was not prominent in our samples as the peak level of the SubPc does not increase significantly while the TTPA peaks are dropping. It suggests the excitons generate by the TTPA layer are more likely to dissociate at the TTPA/SubPc interface than transferring to the SubPc layer. To further confirm the exciton dissociation happened at the TTPA/SubPc interface, we fabricated a solar cell with only TTPA/SubPc layers as the active region and the corresponding J-V cures are shown in Fig. 3(a). It can be noticed that the $\mathrm{J}_{\mathrm{sc}}$ of the device was able to achieve around $1.2 \mathrm{~mA} / \mathrm{cm}^{2}$ with a $\mathrm{V}_{\mathrm{oc}}$ of $0.92 \mathrm{~V}$. By summing up the $\mathrm{J}_{\mathrm{sc}}$ values from the TTPA/SubPc 

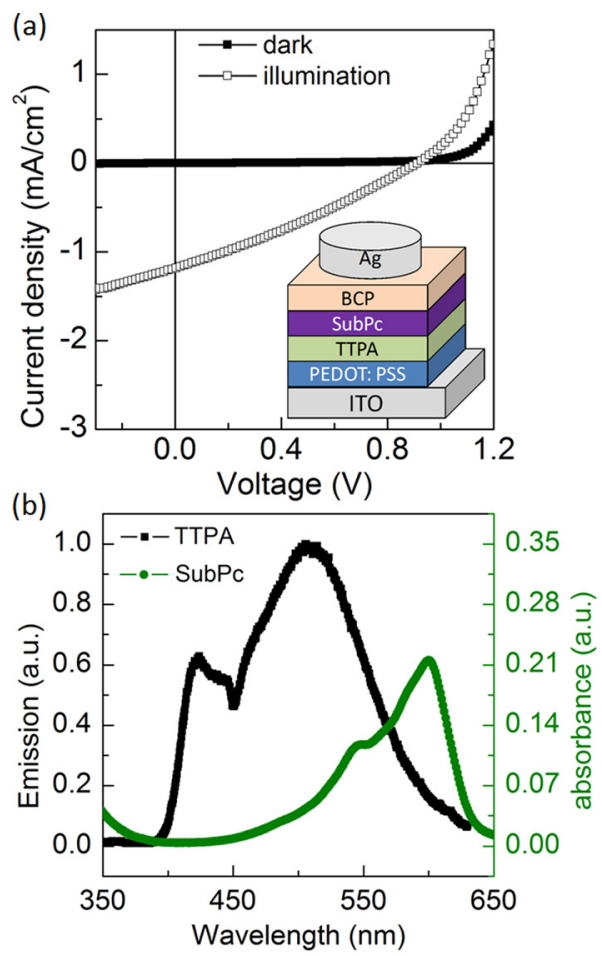

(c)

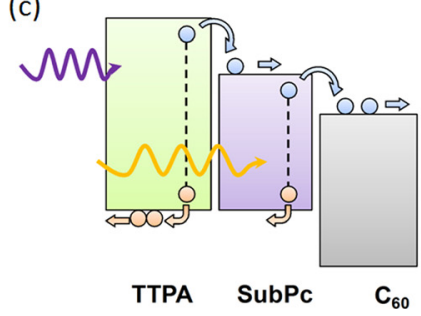

FIG. 3. (Color online) (a) The performance of TTPA/SubPc bilayer device with inserted a schematic diagram of structure (ITO/PEDOT:PSS/ $\operatorname{TTPA}(20 \mathrm{~nm}) / \mathrm{SubPc}(13 \mathrm{~nm}) / \mathrm{BCP}(10 \mathrm{~nm}) / \mathrm{Ag}(100 \mathrm{~nm}))$. (b) The relative value of the emission of TTPA film and the absorbance of SubPc film. (c) The schematic diagram for improvement of photon response by direct charge transfer.

device and the SubPc/C 60 device (unfilled triangle) in Fig. 1 (b), the $\mathrm{J}_{\mathrm{sc}}$ level quantitatively agrees with the multijunction cell. It provides evidence that both interfaces in the multijunction device are contributing to the generation of photocurrent by direct charge transfer in the current OPV structure, although part of the contribution could come from modified electric field distribution. The ineffective energy transfer between the TTPA and SubPc layer could attributed to the weak overlap between their emission and absorption spectra (Fig. 3(b)) which limits the probability of FRET in our current device. ${ }^{2}$ The schematic diagram for the charge transfer process in the multijunction device is shown in Fig. 3(c). The higher energy photons will be absorbed by the TTPA layer and the generated excitons will be dissociated at the TTPA/ SubPc interface for free charge generation. The lower energy photons which are transparent to the TTPA layer will be absorbed by the SubPc for charge generation.

Based on the previous discussion about our experimental findings, we summarized a few key design parameters for the current multijunction OPV. First, the lower bandgap donor material (SubPc in our case) should have reasonable ambipolar carrier transporting properties for efficient charge transfer and phthalocyanine (Pc)-based materials have high potential for such applications. ${ }^{13,14}$ Second, the HOMO levels of the donor materials should be aligned for efficient charge transfer and maintaining the open circuit voltage value. If the HOMO level of higher bandgap donor is larger than the lower bandgap donor, there will be a potential barrier for the hole transport which will further decrease the fill factor and induce S-shape effect in the J-V curves. On the other hand, if the HOMO level of higher bandgap donor is smaller than the low, the $\mathrm{V}_{\mathrm{oc}}$ of the solar cell will be dropped and thus lowering the efficiency of the device. ${ }^{11}$

To conclude, we demonstrated multijunction organic solar cells could improve photon response to the solar spectrum. By introducing the TTPA layer into the active region as an extra donor material, the power conversion efficiency of the devices increased from $1.5 \%$ to $2.2 \%$ while the open circuit voltage remained constant. Different from the previous findings related to Förster resonant energy transfer, the enhancement is correlated to the charges transfer resulting from extra exciton dissociation. Our finding shows than the efficiency enhancement not only requires boarder optical absorption in the active region layers but also the proper design of band alignment for efficient charge transport throughout the device.

J. C. Wang would like to thank J. Ye and W. L. Wong for their help in the measurements of PL and absorption. This work is supported by research grants (Grant Nos. APJ28 and A-PJ73) from Hong Kong Polytechnic University. Funding from HKSAR through UGC grant (Grant No. PolyU 5112/08E) is also acknowledged.

${ }^{1}$ B. E. Hardin, E. T. Hoke, P. B. Armstrong, J. H. Yum, P. Comte, T. Torres, J. M. J. Frechet, M. K. Nazeeruddin, M. Gratzel, and M. D. McGehee, Nature Photon. 3, 406 (2009).

${ }^{2}$ D. Kristina, J. Fang, N. Humphry-Baker, T. Torres, W. T. S. Huck, H. J. Snaith, and R. H. Friend, Nano Lett. 10, 4981 (2010).

${ }^{3}$ W. A. Luhman and R. J. Holmes, Adv. Funct. Mater. 21, 764 (2011).

${ }^{4}$ G. K. Mor, J. Basham, M. Paulose, S. Kim, O. K. Varghese, A. Vaish, S. Yoriya, and C. A. Grimes, Nano Lett. 10, 2387 (2010).

${ }^{5}$ A. Colsmann, J. Junge, C. Kayser, and U. Lemmer, Appl. Phys. Lett. 89, 203506 (2006).

${ }^{6}$ J. Y. Kim, K. Lee, N. E. Coates, D. Moses, T. Q. Nguyen, M. Dante, and A. J. Heeger, Science 317, 222 (2007).

${ }^{7}$ S. Sista, Z. Hong, L. M. Chen, and Y. Yang, Energy Environ. Sci. 4, 1606 (2011).

${ }^{8}$ J. C. Wang, X. C. Ren, S. Q. Shi, C. W. Leung, and P. K. L. Chan, Org. Electron. 12, 880 (2011).

${ }^{9}$ S. Sista, Y. Yao, Y. Yang, M. L. Tang, and Z. Bao, Appl. Phys. Lett. 91, 223508 (2007).

${ }^{10}$ G. Zhang, W. L. Li, B. Chu, L. L. Chen, F. Yan, J. Z. Zhu, Y. R. Chen, and C. S. Lee, Appl. Phys. Lett. 94, 143302 (2009).

${ }^{11}$ Y. Kinoshita, T. Hasobe, and H. Murata, Appl. Phys. Lett. 91, 083518 (2007).

${ }^{12}$ H. Kageyama, H. Ohishi, M. Tanaka, Y. Ohmori, and Y. Shirota, Appl. Phys. Lett. 94, 063304 (2009).

${ }^{13}$ L. S. Hung and M. G. Mason, Appl. Phys. Lett. 78, 3732 (2001).

${ }^{14}$ D. W. I. De Boer, A. F. Stassen, M. F. Craciun, C. L. Mulder, A. Molinari, S. Rogge, and R. F. Morpurgo, Appl. Phys. Lett. 86, 262109 (2005).

${ }^{15}$ See supplementary material at http://dx.doi.org/10.1063/1.3680253 for the devices fabrication and experimental details. 